\title{
Sketch of Togetherness - Episteme temporärer Begegnungen
}

Lea Moro, Alexandra Hennig, Mona De Weerdt

\section{Begegnungen als künstlerische Praxis}

Mit Sketch of Togetherness entwickelt die Choreografin Lea Moro ein serielles performatives Projekt, das die reale körperliche Begegnung von zwei einander unbekannten Personen in den Fokus rückt. Sketch of Togetherness widmet sich den Dynamiken und Möglichkeiten des Sich-Kennenlernens und Begegnens, wird für jeden Spielort neu erarbeitet ${ }^{1}$ und funktioniert nach folgendem Prinzip: Ein"e Akteur"in aus dem Tanz, entweder Lea Moro selbst oder ein*e andere*r Choreograf*in bzw. Tänzer*in/Performer*in, trifft auf eine Person - den Gast - aus einem anderen Berufsfeld, mit dem er*sie einen gemeinsamen Tag verbringt. Ihr Zusammensein wird verhandelt und die Möglichkeiten, einander zu begegnen, auf die Probe gestellt. Der jeweilige Gast bestimmt, wie der Tagesablauf strukturiert wird, schlägt gemeinsame Aktivitäten vor und führt an spezifische Orte seiner"ihrer Wahl. Dabei wird nicht nur die Begegnung mit dem*der Anderen, sondern auch mit dem geografischen Anderswo ins Zentrum gerückt. ${ }^{2}$ Sketch of Togetherness arrangiert somit Begegnungen zwischen zwei Menschen, die vermutlich nie aufeinandertreffen würden, weil sie aus verschiedenen Generationen stammen, unterschiedlichen Berufen nachgehen, an verschiedenen Orten leben und bis

1 Die Sketch-Inszenierungen entstehen jeweils in lokaler Koproduktion mit Theaterinstitutionen oder Festivals. Vgl. die Koproduktionspartner der jeweiligen Sketches auf https:// www.leamoro.com/projects/sketch-of-togetherness/?lang=de

2 Vgl. die Dokumentationen dieser ersten Begegnungen auf https://www.leamoro.com/projects/sketch-of-togetherness/?lang=de 
zum Projektbeginn keine Berührungspunkte miteinander teilen. Im Fokus stehen Fragen wie: Wann und wie können wir uns auf andere Menschen und vielleicht auch auf konträre Wertvorstellungen, Weltanschauungen, Wahrnehmungen und Sichtweisen einlassen? Wie öffnen wir uns für ein neues Thema, einen fremden Gedanken, eine andersartige Welt?

Die Kamera fängt das erstmalige Aufeinandertreffen der beiden Protagonist*innen, deren Gespräche und die zwischen ihnen entstehenden Dynamiken ein. Welche materiellen, physischen und sinnlichen Qualitäten von Begegnung lassen sich beobachten? Bei diesen Begegnungen rücken die Dimensionen des Leiblichen, Sinnlichen und Affektiven in den Fokus, die sich als sicht- und wahrnehmbare Dynamiken zwischen den beiden Personen manifestieren. Sichtbar z.B. in den Körperhaltungen, der Mimik und Gestik oder in der Weise, wie sich die Körper zueinander in Relation setzen, erfahrbar aber auch in den Gesprächsverläufen, den Themen, die adressiert werden, der Art, »wie« etwas gesagt und verhandelt wird (Tonfall, Redetempo, Pausen, Unterbrechungen, Zögern, wer ergreift wann das Wort). Wahrnehmbar darüber hinaus darin, wie die Personen über sich selbst, über ihr eigenes Leben, ihren (Berufs-)Alltag, besondere und prägende Ereignisse etc. erzählen, was sie in diesen Selbsterzählungen von und über sich preisgeben oder auch zurückhalten und wie sie sich zu dem verhalten, was sie in Punkto Geschlecht, sozialer Klasse, Beruf etc. repräsentieren. Gezielt geführt und aufgezeichnet werden im Sinne einer Biografieforschung persönliche Interviews mit Fragen zur Wahrnehmung und Beschreibung des eigenen Körpers, der Persönlichkeit und des Charakters, aber auch mit Fragen zum persönlichen wie gesellschaftlichen Verständnis von togetherness (Miteinander).

\section{Begegnungen in Szene setzen}

Im Anschluss an den gemeinsamen Tagesausflug folgt die Inszenierungsarbeit. Diese beinhaltet:

- Postproduktion:Videomaterial des Tagesausflugs zu dokumentarischen Kurzfilmen schneiden, Audiospuren aus dem Interviewmaterial generieren, Musikauswahl für die Aufführung treffen; 
- Inszenierungsarbeit: Dramaturgie, Szenenabfolge festlegen, Erarbeitung und Proben der Choreografie und der Sprechanteile bzw. Live-Dialoge.

Bei der Inszenierungsarbeit gilt es herauszufinden, wie sich alltagspraktische Erfahrungen und biografische Elemente, solche sens(e)ations also, in einen künstlerisch-performativen Kontext übersetzen, wie sie sich darstellen und vermitteln lassen. Die Inszenierungs- und Probenprozesse konzentrieren sich auf wenige Tage, wobei Proben bedeutet, gemeinsam mit dem jeweiligen Gast die dramaturgische Struktur durchzugehen, die Choreografie zu lernen, Übergänge zu gestalten, räumliche Setzungen vorzunehmen. Dabei entstehen neue Formen der Kollaboration, weil hier auch die jeweiligen Gäste aus anderen Berufsfeldern inszenatorische Ideen beisteuern und den Prozess wie auch die Inszenierung mit ihrem Wissen und ihren Erfahrungen mitgestalten.

Die Sketch-Aufführungen verdichten das während des Tagesausflugs generierte filmische und auditive Material zu Filmsequenzen und Tonspuren und beinhalten performativ-tänzerische, theatrale und dialogische Elemente bzw. Gespräche. Dabei handelt es sich nicht um Wiederholungen der ersten Begegnungen, sondern um Weiterführungen und Ästhetisierungen dieser in einer Live-Situation. Die Aufführungen folgen einer spezifischen dramaturgischen Struktur, die den beiden auftretenden Personen als Gerüst dient und Sicherheit und Orientierung liefert. Dennoch sind sie nicht gänzlich durchgeprobt und lassen Raum für Spontaneität, Ungeplantes und Unvorhersehbares. Was artikuliert sich innerhalb des Aufführungsdispositivs, in diesem gerahmten und doch von gewisser Unvorhersehbarkeit geprägten Raum, zwischen den beiden Sketch-Protagonist*innen?

\section{Sketch of Togetherness \#3: Eine Begegnung zwischen Alberto Veiga und Lea Moro. \\ "It's more difficult to dance than to build a house!«}

Zürich, 06. September 2019. Der Architekt Alberto Veiga erreicht das Tanzhaus Zürich am Morgen der Aufführung von Sketch of Togetherness \#3 um 10.15 Uhr. Während der ersten gemeinsamen Probe der Choreografie, die er von Lea Moro zuvor als Videobotschaft erhalten hatte mit der Aufforderung, 
sie zu lernen, fällt ein bemerkenswerter Satz: »It's more difficult to dance than to build a house!«

Tanzhaus, Studio 2, 20:05 Uhr: Während die Besucher*innen in den Raum strömen, erklingt Monteverdis Lamento della Ninfa. Später erfahren wir, dass dieses klassische Musikstück Alberto Veiga in den letzten fünf Jahren während seiner Arbeitsroutine, genauer als musikalische Untermalung bei der Erstellung seiner morgendlichen To-Do-Liste, begleitet hat. Dies ist eine von vielen persönlichen Offenbarungen, zu denen die zwei Sketch-Akteur"innen sich an diesem Abend vor den Zuschauer"innen hinreißen lassen werden. Über den Zeitraum der Aufführung werden sie sich selbst und einander ein Stück verändert haben. Sie sind das Wagnis eingegangen, sich selbst unbekannt zu werden und mit den Augen des Gegenübers als jemand anderes zu sehen.

Sie setzen zum gemeinsamen Duett an: Nach vorne gestreckte Unterarme, tanzende Finger, die in einem großen Bogen über das Gesicht wandern und sich öffnen (Abb. I) - ein Griff mit der Hand auf die Schulter, eine Kniebeuge - Plié. Der rechte Zeigefinger wird nicht ohne Verlegenheit zur Nase geführt - ihre Blicke begegnen sich (Abb. 2), sie breiten die Arme aus und kreisen fliegend durch den Raum. Das gemeinsam getanzte Duett verbindet die Beiden, es entfaltet durch das Aufeinander-Bezogen-Sein und im Moment des Aufeinander-Achtens verbindendes Potenzial.

Begleitet werden sie von ihren eigenen Stimmen aus dem Off. Innere Monologe, die sich selbst den Spiegel vorhalten. Lea Moro spricht über die ausgeprägten Venen ihrer Arme, die sie von ihrer Mutter habe, als Zeichen von Lebendigkeit und Vitalität. Die Stimme Alberto Veigas ertönt, während er selbst in für ihn sichtlich ungewohnter, wenn auch durchaus tänzerischer Weise um die eigene Achse dreht. Gleichzeitig ertönt von der Tonspur folgender Satz: "My brain is part of my body, too. Diese Bewegungssequenz lebt von den kleinen Unsicherheiten und Fehlern, die nie zu Fehltritten werden, sondern Möglichkeitsräume im Moment der Erfahrung eröffnen: Gemeinsam überschreiten Lea Moro und Alberto Veiga als Kompliz"innen auf unbekanntem Terrain die Stellvertretung ihrer Selbst, sie geben einander Halt und scheuen nicht davor zurück, souverän ins Wanken zu geraten. 

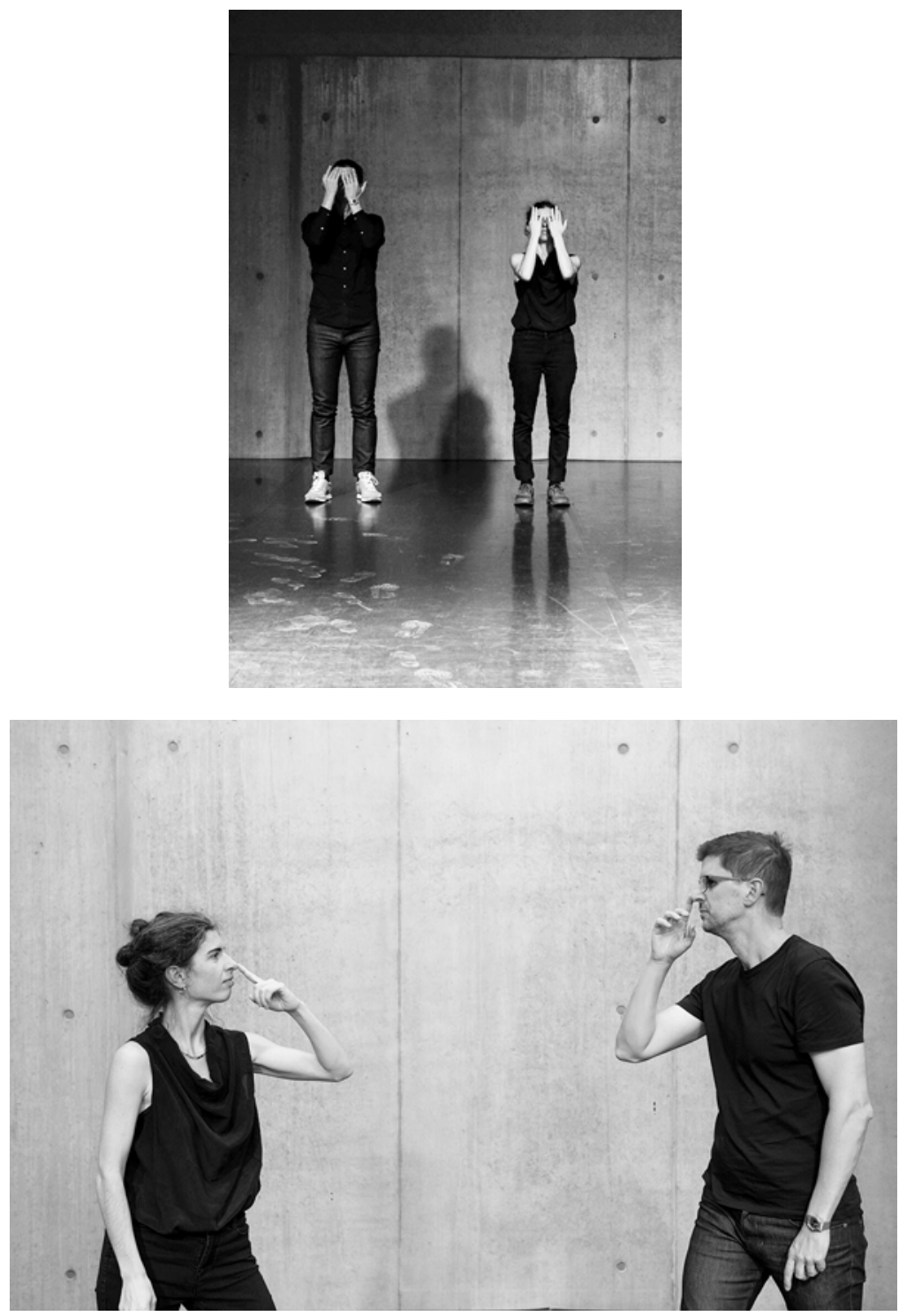

Abb. 1 und Abb. 2: Lea Moro und Alberto Veiga im getanzten Duett während der Aufführung von Sketch of Togetherness \#3, 06.09.2019 Tanzhaus Zürich @ Tina Ruisinger 
Die Beiden begegnen an diesem Abend auch ihren eigenen Professionen auf eine neue Weise: ein Haus zu bauen, das man verlässt, sobald es fertig ist, steht hier der Praxis gegenüber, eine Choreografie zu entwickeln, die sich im Moment der Aufführung beständig transformiert. Als Choreografin und als Architekt teilen sie das Wissen um Prozessualität, Unvorhersehbarkeit, Unabgeschlossenheit und Wandel. Positioniert auf zwei schlichten Rollhockern stellen sie Fragen an ihr Gegenüber, in deren Antworten Momente des Zögerns, kleine Pausen und Abschweifungen verraten, dass sie tatsächlich überlegen: Alberto sagt: »Lea, if you would not be a choreographer, what would you do?« Lea fragt: »Alberto, if you would have the freedom to build anything you want, wherever you want. What would you build?«

Einblicke ihres gemeinsamen Tagesausflugs werden per Videoprojektion eingeblendet. Während sie einander auf der Studiobühne gegenübersitzen, schauen sie sich selbst bei ihrer ersten Begegnung zu: Auf den Rücksitzen eines Taxis begleitete Lea Moro Alberto Veiga ein Jahr zuvor bei seinem allmorgendlichen Weg zum Flughafen. Die Strecke Barcelona - Zürich ist er unzählige Male geflogen. Mit der Eröffnung des Tanzhauses Zürich schließt sich so ein Kapitel seines Alltags. Zwischen dieser Taxifahrt und der Aufführung liegt ein knappes Jahr - die beiden Sketch-Akteur*innen schauen einander und einem schon jetzt historischen, weil früheren Version ihrer Selbst zu. In dem Moment des gemeinsamen Betrachtens der Dokumentation liegt insofern auch eine Rückversicherung: diese Beiden teilen eine gemeinsame Geschichte außerhalb des Theaterraumes, die ihrer ersten Begegnung - der Aufführung ist bereits etwas vorausgegangen.

Dass wir selbst mit der Zeit immer wieder zu jemand anderem werden, dass wir viele Gesichter haben, wird in Sketch of Togetherness auch performativ durch den Akt des Schminkens erfahrbar (Abb. 3). Während Lea Moro einen pinken Streifen über sein Gesicht zieht, bemerkt Alberto Veiga: »I know, that while I am sitting here, the people from my office are recording everything." In diesem Moment deutet sich an, dass in der Begegnung, vor allem aber in der öffentlichen Exposition von sich selbst immer auch buchstäblich etwas auf dem Spiel steht: die aufgelegte Maske erlaubt es, sich selbst preiszugeben und einander dabei gleichzeitig zu Gesicht zu bekommen. Sketch of Togetherness wirkt so als spielerischer Raum, der die Überhöhung des eigenen Selbst in der theatralen Begegnung möglich und performativ erfahrbar macht. Die Akteur*innen stehen sich selbst gegenüber, sie geraten außer-sich. 


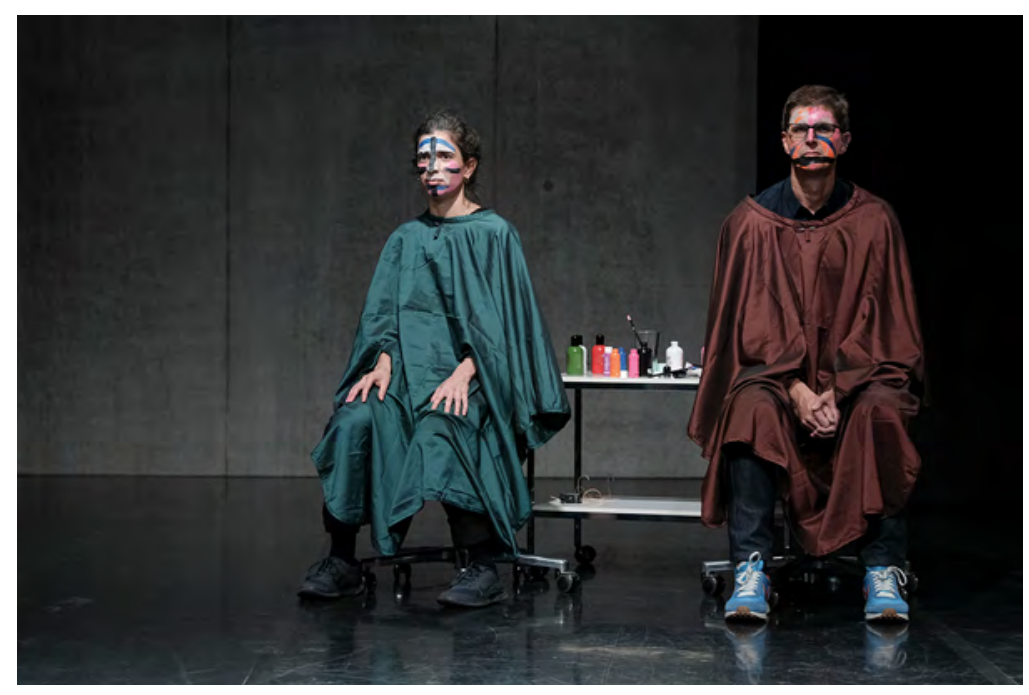

Abb. 3: Lea Moro und Alberto Veiga schminken sich während der Aufführung von Sketch of Togetherness \#3, 06.09.2019 Tanzhaus Zürich (c) Tina Ruisinger

Das erstmalige Aufeinandertreffen aber auch die anschließenden Aufführungen sind somit von sens(e)ation geprägte Momente. Dies in einem doppelten Sinne: Es sind für die beiden Protagonist"innen aufregende Ereignisse, singuläre Momente, die eine Zäsur in deren Alltag setzen und ein Eintauchen in andere Lebensrealitäten erlauben. Während des Tagesausflugs erhalten die Tanzschaffenden Einblicke in andere Berufs- und Lebensrealitäten und andere Alltagsrhythmen, in der Aufführungssituation wiederum begeben sich die jeweiligen Gäste in theatrale Kontexte hinein, exponieren sich öffentlich und erleben sich in einer performativen Situation. Dabei finden sinnliche Prozesse statt, denn für die beiden Sketch-Protagonist*innen rückt die Wahrnehmung des Anderen, das $\mathrm{Zu}$ - und Hinhören in den Fokus. Es eröffnen sich Resonanz- und Reflexionsräume, die Möglichkeiten und Situationen des Miteinanders verhandeln. 


\section{Archiv der Begegnungen}

Die konzeptionelle Ausgangsposition des Projekts erfordert spezifische künstlerische Formen der Archivierung und Vermittlung, um es als serielles Projekt sichtbar zu machen und um die jeweiligen Erfahrungen zu vermitteln und bewahren zu können. Dokumentarisch-künstlerische Kurzfilme halten das erstmalige Aufeinandertreffen zweier sich fremder Personen und die sich zwischen ihnen ergebenden Dynamiken fest. Programmtexte beschreiben das Spezifische jeder Begegnung und die zwischen den beiden Personen aufgeworfenen Themen und verhandelten Fragen. Je nach Gast wird zudem implizit verhandelt, welches Erfahrungs- und Körperwissen in unterschiedlichen Berufsfeldern produziert wird und wie eine Tätigkeit den Körper prägt, definiert oder abnutzt. D.h., es wird evident, dass in jedem Berufsfeld andere Formen von kognitivem, handwerklichem oder physischem Wissen und unterschiedliche Expertisen produziert werden, aber auch, dass jeweils unterschiedliche Sinne oder Fähigkeiten besonders gefordert sind (z.B. das Riechen und Schmecken bei der Winzertätigkeit, das Haptische/ Taktile beim Tanzen, das strategische Denken in der Politik). Somit verweist Sketch of Togetherness auch auf die soziokulturelle Situiertheit und physischsinnliche Materialität von Wissen.

Das Archiv wächst mit jedem Sketch, wobei die Dokumentation auf Lea Moros Homepage ${ }^{3}$ eine nachhaltige Sicherung und breite Sichtbarmachung des Materials sowie eine Reflexion der im Projekt Sketch of Togetherness verhandelten gesellschaftlichen, politischen, sozialen und philosophischen Fragestellungen erlaubt.

\section{Begegnungen mit Ausblick}

Weil sich Begegnungen als dynamisches Prinzip der theoretischen Analyse beständig entziehen, nie ganz in Theoremen oder Denkfiguren aufgehen, immer schon ambivalent, radikal subjektiv und kontextabhängig sind, werden sie bei Sketch of Togetherness zum Gegenstand der künstlerischen (Er-) Forschung. Über die Praxis, einen ganzen Tag mit einer fremden Person

$3 \mathrm{Vgl}$. das wachsende Sketch-Archiv auf https://www.leamoro.com/projects/sketch-of-togetherness/ 
zu verbringen, fordert das Projekt den Luxus der geteilten, unproduktiven und ziellosen Zeit ein. Insofern stellt sich mit Sketch of Togetherness auf einer größeren Ebene auch die Frage, wie es möglich sein kann, einander jenseits von ökonomischen Verwertungslogiken zu begegnen und darüber hinaus Differenzen und Gemeinsamkeiten zu zulassen. Über die jeweiligen Begegnungen transportieren sich Einblicke in unterschiedliche Lebens- und Alltagsrealitäten wie auch Berufswelten, in individuelle Biografien sowie in verschiedene kulturelle, politische und soziale Kontexte. Nicht zuletzt ermöglicht jede Begegnung mit einer fremden Person auch neue Perspektiven auf sich selbst, denn Begegnungen mit anderen Menschen lassen auch das Einnehmen und Revidieren eigener Sichtweisen zu. In Begegnungen liegt ein Potenzial des Zur-Disposition-Stellens von Ansichten und Grundsätzen und vielleicht des Abrückens von festgefahrenen Positionen. Im besten Fall, so die ethische Erwartung, weitet sich durch Begegnung und im Dialog der emotionale und kognitive Horizont des Einzelnen.

Sketch of Togetherness versteht sich insofern als ein prozessuales, niemals abgeschlossenes Großprojekt, das durch mehrere Serien (die jeweiligen Begegnungen) auch auf größere politische, kulturelle, gesellschaftliche und intersubjektive Kontexte verweist und Aufschluss über Begegnung »an sich" liefern kann. Temporäre Begegnungen wirken hier als Episteme für das künstlerische Schaffen weit über die einzelnen Sketches hinaus. Zwischenmenschliche Beziehungen, die Wahrnehmung des eigenen Selbst sowie des*der Anderen, das Aufeinandertreffen verschiedener Alltagsrealitäten und Lebenswelten, sind Bestandteile des Projekts, das choreografisch und dramaturgisch außerordentlichen sens(e)ations nachspürt.

\section{Literatur}

https://www.leamoro.com/projects/sketch-of-togetherness/?lang=de [online] [06.01.2020] 
